\title{
Dense Semi-Rigid Scene Flow Estimation from RGBD images *
}

\author{
Julian Quiroga ${ }^{1,3}$, Thomas Brox ${ }^{2}$, Frédéric Devernay ${ }^{1}$, and James Crowley ${ }^{1}$ \\ 1 PRIMA team, INRIA Grenoble, France \\ firstname.lastname@inria.fr \\ 2 Department of Computer Science, University of Freiburg, Germany \\ brox@cs.uni-freiburg.de \\ 3 Departamento de Electrónica, Pontificia Universidad Javeriana, Colombia \\ quiroga.j@javeriana.edu.co
}

\begin{abstract}
Scene flow is defined as the motion field in 3D space, and can be computed from a single view when using an RGBD sensor. We propose a new scene flow approach that exploits the local and piecewise rigidity of real world scenes. By modeling the motion as a field of twists, our method encourages piecewise smooth solutions of rigid body motions. We give a general formulation to solve for local and global rigid motions by jointly using intensity and depth data. In order to deal efficiently with a moving camera, we model the motion as a rigid component plus a non-rigid residual and propose an alternating solver. The evaluation demonstrates that the proposed method achieves the best results in the most commonly used scene flow benchmark. Through additional experiments we indicate the general applicability of our approach in a variety of different scenarios.
\end{abstract}

Keywords: motion, scene flow, RGBD image

\section{Introduction}

The 3D motion field of a scene is useful for several computer vision applications, such as action recognition, interaction, or 3D modeling on nonrigid objects. One group of methods uses a stereo or multi-view camera system, where both scene flow and depth are estimated from the images, while a second group uses RGBD images as input. In the latter case, the depth given by the sensor may be used directly for scene flow estimation. In this paper, we present a new formulation for this second group.

The two main questions for scene flow estimation from RGBD images are: (a) how to fully exploit both sources of data, and (b) which motion model should be used to compute a confident scene flow. When both intensity and depth are

\footnotetext{
^ This work was supported by a collaborative research program between Inria Grenoble and University of Freiburg. We gratefully acknowledge partial funding by CMIRA 2013 (Region Rhône-Alpes), GDR ISIS (CNRS), and COLCIENCIAS (Colombia).
} 
provided, there is not a consensus on how to combine these two sources of information most effectively. A straightforward approach would compute the optical flow from RGB images and infer the scene flow from the depth data. It is also possible to generate colored $3 \mathrm{D}$ point clouds and try to find out the $3 \mathrm{D}$ motion vectors consistent with the input data. However, it is not mandatory to explicitly represents points in 3D to solve for the scene flow: the scene structure can be represented in the image domain, where color and depth data can be coupled using a projective function. This way, depth changes influence the motion in the image domain and consistency constraints can be formulated jointly over the color and depth images. This is the approach we follow in this work. It gives us access to some of the powerful tools developed for 2D motion estimation.

The second question has been addressed less. Scene flow estimation using intensity and depth is an ill-posed problem and regularization is needed. Usually, the $3 \mathrm{D}$ motion vector of each point is solved to minimize color and intensity constraints in a data term and the whole 3D motion field is regularized to get spatially smooth solutions while preserving discontinuities. Since depth data is available, a weighted regularization can be used to preserve motion discontinuities along depth edges, where independent motions are probable to appear. However, solving for a piecewise smooth solution of the 3D motion field may not be the best choice for some motions of interest. For example, a 3D rotation of a rigid surface induces a great variety of $3 \mathrm{D}$ motions that are hardly well regularized by such an approach. A similar issue occurs when the RGBD sensor is moving with respect to the scene.

In this work, we take advantage of the fact that most real scenes can be well modeled as locally or piecewise rigid, i.e., the scene is composed of 3D independently rigid components. The main contribution of this paper is the definition of an over-parametrized framework for scene flow estimation from RGBD images. We model the scene flow as a vector field of rigid body motions. This representation helps the regularization process which, instead of directly penalizing variations in the $3 \mathrm{D}$ motion field, encourages piecewise smooth solutions of rigid motions. Moreover, the proposed rigid body approach can be constrained in the image domain to fully exploit intensity and depth data. This formulation is flexible enough to support different data constraints and regularization strategies, and it can be adapted to more specialized problems. By using the same general framework, it is possible to model the scene flow as a global rigid motion plus a non-rigid residual, which is particularly useful when estimating the motion of deformable objects in conjunction with a moving camera.

\section{Related work}

Scene flow was first introduced by Vedula [18] as the 3D motion field of the scene. Since this seminal work several approaches have been proposed to compute the 3D motion field. If a stereo or multi-view camera system is available, the scene flow can be computed by enforcing consistency with the observed optical flows [18], by a decoupled [21] or joint $[1,8]$ estimation of structure and motion, 
or by assuming a local rigidity of the scene [19]. In this work we assume that a depth sensor is available and the estimation of the structure is not needed. The first work using intensity and depth was by Spies et al. [16], where the optical flow formulation by Horn and Schunck [7] is extended to include depth data. In this approach, depth data is used simply as an additional channel in the variational formulation of the optical flow. The range flow is estimated using the observed data and enforced to be smooth. However, the scene is assumed to be captured by an orthographic camera and there is no coupling between optical and range flows. The coupling issue can be solved as in [10], where the scene flow is directly solved using the depth data to constrain the $3 \mathrm{D}$ motion in the image domain. However in that work, the depth data is not fully exploited since there is no range flow constraint. Similar to [16], this method suffers from the early linearization of the constancy constraints and from over-smoothing along motion boundaries because of the $L^{2}$-regularization. Quiroga et al. [12] define a 2D warping function to couple image motion and 3D motion, allowing for a joint local constraint of the scene flow on intensity and depth data. Although the method is able to deal with large displacements, it fails on untextured regions and more complex motions, such as rotations.

In order to solve for dense scene flow, a regularization procedure is required. Usually, the 3D motion field is assumed to be piecewise smooth, and total variation (TV) is used as regularizer. The work by Herbst [6] follows this idea, but as [16], it lacks a coupling between optical and range flows, and the regularization is done on the optical flow rather than on the scene flow. In [13], a variational extension of [12] is presented. A weighted TV is applied on each component of the $3 \mathrm{D}$ motion field, aiming to preserve motion discontinuities along depth edges.

All these methods assume spatial smoothness of the scene flow, which is a reasonable assumption for translational motions but not for rotations. Under a rotation, even close scene points present different $3 \mathrm{D}$ motions. In case of a moving camera the regularization of the motion field can be a challenge. In this work, we use an over-parametrization of the scene flow, where each scene point is allowed to follow a rigid body motion. This way, the regularization can be done on a field of rigid motions, favoring piecewise solutions, which is a better choice for real scenes. A similar idea is presented in [14], where a regularization of a field of rigid motions is proposed. Our approach differs from that work in three ways. First, we use a more compact representation of the rigid-body motion via the 6 -parameter twist representation, instead of a $\mathbb{R}^{12}$ embedding. Second, our approach solves and regularizes the rigid motion field at the same time. Finally, we decouple the regularization of the rotational and translational fields, which simplifies the optimization and allows the use of different TV strategies on each field. Similar to [12], we use a depth-based weighting function to avoid penalization along surface discontinuities.

In an alternative approach, Hadfield and Bowden [5] estimate the scene flow using particle filtering in a $3 \mathrm{D}$ colored point cloud representation of the scene. In that approach, a large set of motion hypotheses must be generated and tested for each $3 \mathrm{D}$ point, leading to high computational costs. We rather use the $2 \mathrm{D}$ 
parametrization provided by RGBD sensors to formulate an efficient 3D motion exploration. As done in [12], we define a warping function to couple the twist motion and the optical flow. A very similar warp is presented in [9] to solve for a global rigid motion from RGBD images. In our approach, we use the warping function to locally constrain the rigid motion field in the image domain. The global rigid motion estimation can be seen as a particular case of the proposed formulation. Moreover, unlike [9], we define a depth consistency constraint to fully exploit both sources of data. Thus, we can solve for the local twist motion that best explains the observed intensity and depth data, gaining robustness under noise. The local solver, in conjunction with the TV regularization of the twist field, provides an adjustable combination between local and piecewise rigidity.

As we present in the experiments, this framework is flexible enough to estimate a global rigid body motion or to solve for a general 3D motion field in challenging setups. This way, we are able to model the motion of the scene as a global rigid motion and a non-rigid residual.

\section{Scene motion model}

We parameterize every visible $3 \mathrm{D}$ point into the image domain $\boldsymbol{\Omega} \subset \mathbb{R}^{2}$. The projection $\pi: \mathbb{R}^{3} \rightarrow \mathbb{R}^{2}$ maps a $3 \mathrm{D}$ point $\mathbf{X}=(X, Y, Z)$ onto a pixel $\mathbf{x}=(x, y)$ on $\Omega$ by:

$$
\pi(\mathbf{X})=\left(f_{x} \frac{X}{Z}+c_{x}, f_{y} \frac{Y}{Z}+c_{y}\right)^{T},
$$

where $f_{x}$ and $f_{y}$ are the focal lengths of the camera and $\left(c_{x}, c_{y}\right)$ its principal point. The inverse projection $\pi^{-1}: \mathbb{R}^{2} \times \mathbb{R} \rightarrow \mathbb{R}^{3}$ back-projects an image point to the 3D space for a given depth $z$, as follows:

$$
\pi^{-1}(\mathbf{x}, z)=\left(z \frac{x-c_{x}}{f_{x}}, z \frac{y-c_{y}}{f_{y}}, z\right)^{T} .
$$

We will use $\mathbf{X}$ or $\pi^{-1}(\mathbf{x}, z)$ as the $3 \mathrm{D}$ representation of image point $\mathbf{x}$.

The scene flow $\mathbf{v}(\mathbf{x}): \boldsymbol{\Omega} \rightarrow \mathbb{R}^{3}$ is defined as the $3 \mathrm{D}$ motion field describing the motion of every visible $3 \mathrm{D}$ point between two time steps accordingly

$$
\mathbf{X}_{t+1}=\mathbf{X}_{t}+\mathbf{v}\left(\mathbf{x}_{t}\right) \text {. }
$$

\subsection{Twist motion field}

Instead of directly representing the elements of the motion field as $3 \mathrm{D}$ vectors, we use an over-parametrized model to describe the motion of every point as a rigid transformation. The group action of a rigid body transformation can be written as $T(\mathbf{X})=\mathbf{R X}+\mathbf{t}$, where $\mathbf{t} \in \mathbb{R}^{3}$ is a translation, and $\mathbf{R} \in S O(3)$ is a rotation matrix. Using homogeneous coordinates, a $3 \mathrm{D}$ point $\tilde{\mathbf{X}}=(\mathbf{X}, 1)^{T}$ is transformed into $\tilde{\mathbf{X}}^{\prime}$ accordingly to

$$
\tilde{\mathbf{X}}^{\prime}=\mathbf{G} \tilde{\mathbf{X}}, \quad \text { with } \quad \mathbf{G}=\left(\begin{array}{cc}
\mathbf{R} & \mathbf{t} \\
\mathbf{0}_{1 \times 3} & 1
\end{array}\right) \in S E(3) .
$$


Since $\mathbf{G}$ has only 6 degrees of freedom, a more convenient and compact representation is the 6-parameter twist. Every rigid motion can be described as a rotation around a 3D axis $\omega=\left(\omega_{X}, \omega_{Y}, \omega_{Z}\right)^{T}$ and a translation $\tau=\left(\tau_{x}, \tau_{y}, \tau_{z}\right)^{T}$ along this axis. Therefore it can be shown that for any arbitrary $\mathbf{G} \in S E(3)$ there exists an equivalent $\xi \in \mathbb{R}^{6}$ twist representation. A twist $\xi=(\tau, \omega)$ can be converted into the $\mathbf{G}$ representation with the following exponential map:

$$
\mathbf{G}=e^{\hat{\xi}}=\mathbf{I}+\hat{\xi}+\frac{(\hat{\xi})^{2}}{2 !}++\frac{(\hat{\xi})^{3}}{3 !}+\cdots
$$

where

$$
\hat{\xi}=\left(\begin{array}{cc}
\hat{\omega} & \tau \\
\mathbf{0}_{1 \times 3} & 1
\end{array}\right) \text {, with } \hat{\omega}=\left(\begin{array}{ccc}
0 & -\omega_{Z} & \omega_{Y} \\
\omega_{Z} & 0 & -\omega_{X} \\
-\omega_{Y} & \omega_{X} & 0
\end{array}\right) .
$$

Correspondingly, for each $\mathbf{G} \in S E(3)$ there exist a twist representation given by the logarithmic map via $\xi=\log (\mathbf{G})$; see [11] for more details. The motion of the scene is embedded in a twist motion field $\xi(\mathbf{x}): \Omega \rightarrow \mathbb{R}^{6}$, where the motion of every 3D point between two time steps is given by:

$$
\tilde{\mathbf{X}}_{t+1}=e^{\hat{\xi}\left(\mathbf{x}_{t}\right)} \tilde{\mathbf{X}}_{t} .
$$

\subsection{Twist motion on the image}

For every time $t$ the scene is registered as an RGBD image $\mathbf{S}_{t}(\mathbf{x})=\left\{I_{t}(\mathbf{x}), Z_{t}(\mathbf{x})\right\}$ where images $I(\mathbf{x})$ and $Z(\mathbf{x})$ provide color and depth, respectively, for every point $\mathbf{x} \in \boldsymbol{\Omega}$. Under the action of a twist $\xi$ between $t$ and $t+1$, the motion of a $3 \mathrm{D}$ point induces an image flow $(u, v)$. We define the warping function $\mathbf{W}(\mathbf{x}, \xi): \mathbb{R}^{2} \times \mathbb{R}^{6} \rightarrow \mathbb{R}^{2}$ that maps each non-occluded pixel onto its new location after the rigid motion. The warping function is defined as:

$$
\mathbf{W}(\mathbf{x}, \xi)=\pi\left(e^{\hat{\xi}} \tilde{\mathbf{X}}\right), \quad \text { with } \quad \tilde{\mathbf{X}}=\left(\begin{array}{c}
\pi^{-1}(\mathbf{x}, Z(\mathbf{x})) \\
1
\end{array}\right) .
$$

Let $S^{k}(\mathbf{x})$ be a component of the RGBD image (e.g. brightness or depth) evaluated at $\mathbf{x} \in \boldsymbol{\Omega}$ and let $\rho_{S^{k}}(\mathbf{x}, \xi)$ be a robust error function between $S_{t}^{k}(\mathbf{x})$ and $S_{t+1}^{k}(\mathbf{W}(\mathbf{x}, \xi))$. Every twist motion vector $\xi$ is solved to minimize one or several consistency functions $\rho_{S^{k}}(\mathbf{x}, \xi)$. Optimization problems on manifolds such as $S E(3)$ can be solved by calculating incremental steps in the tangent space to the manifold. Considering that an initial estimate $\xi$ is known, the goal at each optimization step is to find the increment $\Delta \xi$ which (approximately) minimizes $\rho_{S^{k}}(\mathbf{x}, \xi)$. Since $S E(3)$ is a Lie group (not an Euclidean space) with the composition as operation, the exponential and logarithmic functions are used to update the current estimate $\xi$ with the new increment $\Delta \xi$ according to $\xi \leftarrow \log \left(e^{\hat{\Delta \xi}} e^{\hat{\xi}}\right)$ [11]. The iterative solution requires a linearization of the warping function. Given the initial estimate $\xi$, the image point $\mathbf{x}$ becomes $\mathbf{x}_{\xi}=\mathbf{W}(\mathbf{x}, \xi)$ and the warping function satisfies:

$$
\mathbf{W}\left(\mathbf{x}, \log \left(e^{\hat{\Delta \xi}} e^{\hat{\xi}}\right)\right)=\mathbf{W}\left(\mathbf{x}_{\xi}, \Delta \xi\right)=\mathbf{x}_{\xi}+\delta \mathbf{x}\left(\mathbf{x}_{\xi}, \Delta \xi\right),
$$


where $\delta \mathbf{x}\left(\mathbf{x}_{\xi}, \Delta \xi\right)$ is the image flow induced by the increment $\Delta \xi$, which is given by $\delta \mathbf{x}\left(\mathbf{x}_{\xi}, \Delta \xi\right)=\pi\left(e^{\hat{\Delta} \xi} \tilde{\mathbf{X}}_{\xi}\right)-\pi\left(\tilde{\mathbf{X}}_{\xi}\right)$, with $\tilde{\mathbf{X}}_{\xi}=\left(\pi^{-1}\left(\mathbf{x}_{\xi}, Z\left(\mathbf{x}_{\xi}\right)\right), 1\right)^{T}$. Assuming a small rotation increment, the exponential function is approximated as $e^{\hat{\Delta \xi}} \approx$ $\mathbf{I}+\hat{\Delta} \xi$. Therefore for a small increment $\Delta \xi$ the warping function can be well approximated by the following linear version:

$$
\mathbf{W}\left(\mathbf{x}, \log \left(e^{\hat{\Delta} \xi} e^{\hat{\xi}}\right)\right)=\mathbf{W}(\mathbf{x}, \xi)+\mathbf{J}\left(\mathbf{x}_{\xi}\right) \Delta \xi,
$$

where $\mathbf{J}\left(\mathbf{x}_{\xi}\right)$ is the Jacobian matrix, given by:

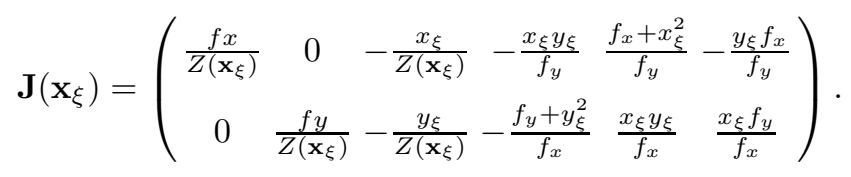

\section{Scene flow formulation}

Given two pairs of RGBD images $\left\{I_{1}, Z_{1}\right\}$ and $\left\{I_{2}, Z_{2}\right\}$ the goal is to solve for the scene flow field that best explains the observed data. Due to vanishing gradients, the aperture problem and outliers, this motion computation is an illposed problem that cannot be solved independently for each point. Therefore, a smoothness in the motion field must be assumed. In our formulation, we consider only spatial smoothness, but it can be extended to temporal smoothness. In general, we solve for the twist field $\xi$ minimizing the following energy:

$$
E(\xi)=E_{D}(\xi)+\alpha E_{S}(\xi)
$$

where the data term $E_{D}(\xi)$ measures how consistent is the estimated twist-based model with the observed color and depth data, and the smoothness term favors piecewise smooth fields while preserving discontinuities.

\subsection{Data term}

The warping function (8) enables the formulation of consistency constraints for the twist field in the image domain. Using the gray value image we define a brightness constancy assumption:

$$
I_{2}(\mathbf{W}(\mathbf{x}, \xi))=I_{1}(\mathbf{x})
$$

and a gradient constancy assumption:

$$
I_{2}^{g}(\mathbf{W}(\mathbf{x}, \xi))=I_{1}^{g}(\mathbf{x}) .
$$

where $I^{g}(\mathbf{x})=|\nabla I(\mathbf{x})|$. The gradient assumption reduces the effect of brightness changes. We use the gradient magnitude since it is invariant to rotation. The estimated twist motion should satisfy these constraints for most points. 
On the other hand, the surface changes under the twist action. This variation must be consistent with the observed depth data. Therefore we define a depth variation constraint given by:

$$
Z_{2}(\mathbf{W}(\mathbf{x}, \xi))=Z_{1}(\mathbf{x})+\delta_{Z}(\mathbf{x}, \xi)
$$

where $\delta_{Z}(\mathbf{x}, \xi)$ is the depth variation induced on the $3 \mathrm{D}$ point $\pi^{-1}\left(\mathbf{x}, Z_{1}(\mathbf{x})\right)$ by the twist $\xi$, obtained from the third component of the $3 \mathrm{D}$ vector:

$$
\delta_{\mathbf{3} \mathbf{D}}(\mathbf{x}, \xi)=\left(e^{\hat{\xi}}-\mathbf{I}_{4 \times 4}\right) \tilde{\mathbf{X}} \quad \text { with } \quad \tilde{\mathbf{X}}=\left(\begin{array}{c}
\pi^{-1}\left(\mathbf{x}, Z_{1}(\mathbf{x})\right) \\
1
\end{array}\right)
$$

This equation enforces the consistency between the motion captured by the depth sensor and the estimated motion.

Without regularization, equations (13), (14) and (15) alone are not sufficient to constrain the twist motion for a given point since there is an infinite number of twists that satisfy these constraints. However, real scenes are locally rigid and it is possible to solve for a local twist explaining the observed motion. We formulate the twist motion estimation as a local least-square problem by writing the data term as follows:

$$
E_{D}(\xi)=\sum_{\mathbf{x}} \sum_{\mathbf{x}^{\prime} \in N_{\mathbf{x}}} \Psi\left(\rho_{I}^{2}\left(\mathbf{x}^{\prime}, \xi(\mathbf{x})\right)+\gamma \rho_{g}^{2}\left(\mathbf{x}^{\prime}, \xi(\mathbf{x})\right)\right)+\lambda \Psi\left(\rho_{Z}^{2}\left(\mathbf{x}^{\prime}, \xi(\mathbf{x})\right)\right)
$$

where $N_{\mathbf{x}}$ is an image neighborhood centered on $\mathbf{x}$, and with the brightness, gradient and depth residuals, respectively given by:

$$
\begin{aligned}
\rho_{I}(\mathbf{x}, \xi) & =I_{2}(\mathbf{W}(\mathbf{x}, \xi))-I_{1}(\mathbf{x}) \\
\rho_{g}(\mathbf{x}, \xi) & =I_{2}^{g}(\mathbf{W}(\mathbf{x}, \xi))-I_{1}^{g}(\mathbf{x}) \\
\rho_{Z}(\mathbf{x}, \xi) & =Z_{2}(\mathbf{W}(\mathbf{x}, \xi))-\left(Z_{1}(\mathbf{x})+\delta_{Z}(\mathbf{x}, \xi)\right) .
\end{aligned}
$$

Constant $\gamma$ balances brightness and gradient residuals, while $\lambda$ weights intensity and depth terms. We use the robust norm $\Psi\left(s^{2}\right)=\sqrt{s^{2}+\varepsilon^{2}}$, which is a differentiable approximation of the $L^{1}$ norm, to cope with outliers due to occlusion and non-rigid motion components in $N_{\mathbf{x}}$.

\subsection{Smoothness term}

A twist motion field $\xi$ can be decomposed into a rotational field $\omega$, with $e^{\hat{\omega}}: \Omega \rightarrow$ $S O(3)$, and a $3 \mathrm{D}$ motion field $\tau: \Omega \rightarrow \mathbb{R}^{3}$. Since they are decorrelated by nature, we regularize each field independently using weighted Total Variation (TV), which allows piecewise smooth solutions while preserving motion discontinuities.

Regularization of the fields $\omega$ and $\tau$ poses different challenges. Elements of $\tau$ lie in the Euclidean space $\mathbb{R}^{3}$ and the problem corresponds to a vector-valued function regularization. Different TV approaches can be used to regularize $\tau$, as described in [4]. Particularly the channel-by-channel $L^{1}$ norm has been successfully used for optical flow [22]. We define the weighted TV of $\tau$ as:

$$
\mathbf{T V}_{c}(\tau)=\sum_{\mathbf{x}} c(\mathbf{x})\|\nabla \tau(\mathbf{x})\|
$$


where $\|\nabla \tau\|:=\left|\nabla \tau_{X}\right|+\left|\nabla \tau_{Y}\right|+\left|\nabla \tau_{Z}\right|$ and $c(\mathbf{x})=e^{-\beta\left|\nabla Z_{1}(\mathbf{x})\right|^{2}}$. The weighting function $c$ helps preserve motion discontinuities along edges of the $3 \mathrm{D}$ surface. Moreover, the $L^{1}$ norm can be replaced by the Huber norm [23] to reduce the staircasing effect. Efficient solvers for both norms are presented in [3].

Elements of $\omega$ are rotations in the Lie group $S O(3)$ embedded in $\mathbb{R}^{3}$ through the exponential map, and the regularization has to be done on this manifold. In order to apply a TV regularization, a notion of variation should be used for elements of $S O(3)$. Given two points $e^{\hat{\omega}_{1}}, e^{\hat{\omega}_{2}} \in S O(3)$ the residual rotation can be defined as $e^{-\hat{\omega}_{1}} e^{\hat{\omega}_{2}}$. The product in logarithmic coordinates can be expressed as $e^{\hat{\omega}_{1}} e^{\hat{\omega}_{1}}=e^{\mu\left(\hat{\omega}_{1}, \hat{\omega}_{2}\right)}$, where the mapping $\mu$ can be expanded in a Taylor series around the identity, using the Baker-Campbell-Hausdorff formula:

$$
\mu\left(\hat{\omega}_{1}, \hat{\omega}_{2}\right)=\hat{\omega}_{1}+\hat{\omega}_{2}+\frac{1}{2}\left[\hat{\omega}_{1}, \hat{\omega}_{2}\right]+O\left(\left|\left(\hat{\omega}_{1}, \hat{\omega}_{2}\right)\right|^{3}\right),
$$

where $[\cdot, \cdot]$ is the Lie bracket in $s o(3)$. Close to the identity, $(22)$ is well approximated by its first-order terms, so that for small rotations the variation measure can be defined as the matrix subtraction in $s o(3)$, or equivalently, as a vector difference for the embedding in $\mathbb{R}^{3}$. Accordingly, the derivative matrix $\mathbf{D} \omega:=\left(\nabla \omega_{X}, \nabla \omega_{Z}, \nabla \omega_{Z}\right)^{T}: \boldsymbol{\Omega} \rightarrow \mathbb{R}^{3 \times 2}$ approximates the horizontal and vertical point-wise variations of $\omega$ on the image. Following [4], we define the TV as the sum over the largest singular value $\sigma_{1}$ of the derivative matrix:

$$
\mathbf{T V}_{\sigma}(\omega)=\sum_{\mathbf{x}} c(\mathbf{x}) \sigma_{1}(\mathbf{D} \omega(\mathbf{x}))
$$

This TV approach supports a common edge direction for three components, which is a desirable properties for the regularization of the field of rotations. Moreover, deviations are less penalized with respect to other measures (e.g. Frobenius norm [14]) and efficient solvers are available. However, this TV definition approximates the real structure of the manifold yielding to a biased measures far from the identity. The more the rotation is away from the identity, the more its variations in $S O(3)$ are penalized as is shown hereinafter. Given two rotations $\omega_{1}=\theta_{1} \overleftarrow{\omega_{1}}$ and $\omega_{2}=\theta_{2} \overleftarrow{\omega_{2}}$, with $\theta$ the angle and $\overleftarrow{\omega}$ the unitary axis vector of the rotation, and writing $\theta_{2}=\theta_{1}+\delta \theta$, leads to $\omega_{2}-\omega_{1}=\theta_{1}\left(\overleftarrow{\omega_{2}}-\overleftarrow{\omega_{1}}\right)-\delta \theta \overleftarrow{\omega_{2}}$. This linearly dependent penalization usually is not a problem, since large rotations imply larger motion on the image. Thus, a stronger regularization can be reasonable. Moreover, large rotation caused by a global motion of the scene or the camera can be optimized separately and compensated, as we show in Sec. 4.4. Optionally, this over-penalization can be removed by expressing each rotation as $\omega=\theta \overleftarrow{\omega}$ and applying a vectorial TV on $\overleftarrow{\omega}$ and a scalar TV on $\theta$. In our approach, the full smoothing term is given by:

$$
E_{S}(\xi)=\mathbf{T V}_{c}(\tau)+\mathbf{T} \mathbf{V}_{\sigma}(\omega)
$$




\subsection{Optimization}

The proposed energy (12) is minimized by decomposing the optimization into two simpler problems. We use the variable splitting method [20] with auxiliary variable $\chi$, and the minimization problem becomes:

$$
\min _{\xi, \chi} E_{D}(\xi)+\frac{1}{2 \kappa} \sum_{\mathbf{x}}|\xi(\mathbf{x})-\chi(\mathbf{x})|^{2}+\alpha E_{S}(\chi),
$$

where $\kappa$ is a small numerical variable. Note that the linking term between $\xi$ and $\chi$ is the distance on the tangent space at the identity in $S E(3)$. The solution of (25) converges to that of (12) as $\kappa \rightarrow 0$. Minimization of this energy is performed by alternating the two following optimization problems:

i. For fixed $\chi$, estimate $\xi$ that minimizes (25). This optimization problem can be solved point-wise by minimizing:

$$
\frac{1}{2 \kappa}|\xi-\chi|^{2}+\sum_{\mathbf{x}^{\prime} \in N_{\mathbf{x}}} \Psi\left(\rho_{I}^{2}+\gamma \rho_{g}^{2}\right)+\lambda \Psi\left(\rho_{Z}^{2}\right),
$$

where the parameters $\left(\mathbf{x}^{\prime}, \xi\right)$ are considered implicit. This energy can be linearized around an initial estimate $\xi$, using a first-order Taylor series expansion:

$$
\begin{array}{r}
\frac{1}{2 \kappa}\left|\log \left(e^{\Delta \hat{\xi}} e^{\hat{\xi}}\right)-\chi\right|^{2}+\sum_{\mathbf{x}^{\prime} \in N_{\mathbf{x}}} \Psi\left(\left|\rho_{I}+\mathbf{I}_{\mathbf{x}} \mathbf{J} \Delta \xi\right|^{2}+\gamma\left|\rho_{G}+\mathbf{I}_{\mathbf{x}}^{g} \mathbf{J} \Delta \xi\right|^{2}\right) \\
+\lambda \Psi\left(\left|\rho_{Z}+\left(\mathbf{Z}_{\mathbf{x}} \mathbf{J}-\mathbf{K}\right) \Delta \xi\right|^{2}\right) .
\end{array}
$$

where $\mathbf{I}_{\mathbf{x}}, \mathbf{I}_{\mathbf{x}}^{g}$ and $\mathbf{Z}_{\mathbf{x}}$ are the row vector gradients of $\mathbf{I}_{2}(\mathbf{x}), \mathbf{I}_{2}^{g}(\mathbf{x})$ and $\mathbf{Z}_{2}(\mathbf{x})$, respectively, and $\mathbf{J}$ is the Jacobian (11) of the warp, all evaluated at $\mathbf{x}_{\xi}=$ $\mathbf{W}(\mathbf{x}, \xi)$. The $1 \times 6$ vector $\mathbf{K}$ is defined as $\mathbf{K}=\mathbf{D}\left(\left[\mathbf{X}_{\xi}\right]_{\times} \mid \mathbf{I}_{3 \times 3}\right)$ with $\mathbf{D}=(0,0,1)$ isolating the third component and $[\cdot]_{\times}$the cross product matrix. Finding the minimum of (27) requires an iterative approach. Taking the partial derivative with respect to $\Delta \xi$ and setting it to zero, the increment $\Delta \xi$ can be computed as:

$$
\begin{aligned}
\Delta \xi=-\mathbf{H}^{-1}\left[\sum _ { \mathbf { x } ^ { \prime } \in N _ { \mathbf { x } } } \left\{\Psi^{\prime}\left(\rho_{I}^{2}+\gamma \rho_{G}^{2}\right)\left[\left(\mathbf{I}_{\mathbf{x}} \mathbf{J}\right)^{T} \rho_{I}+\gamma\left(\mathbf{I}_{\mathbf{x}}^{g} \mathbf{J}\right)^{T} \rho_{G}\right]\right.\right. \\
\left.\left.+\lambda \Psi^{\prime}\left(\rho_{Z}^{2}\right)\left(\mathbf{Z}_{\mathbf{x}} \mathbf{J}-\mathbf{K}\right)^{T} \rho_{Z}\right\}+\frac{1}{\kappa} \log \left(\left(e^{\hat{\xi}}\right)^{-1} e^{\hat{\chi}}\right)\right],
\end{aligned}
$$

where $\Psi^{\prime}$ is the derivative of the robust norm, which is evaluated at the current estimate $\xi$. The $6 \times 6$ matrix $\mathbf{H}$ is the Gauss-Newton approximation of the Hessian matrix and is given by:

$$
\begin{aligned}
& \mathbf{H}=\sum_{\mathbf{x}^{\prime} \in N_{\mathbf{x}}} \Psi^{\prime}\left(\rho_{I}^{2}+\gamma \rho_{G}^{2}\right) {\left[\left(\mathbf{I}_{\mathbf{x}} \mathbf{J}\right)^{T}\left(\mathbf{I}_{\mathbf{x}} \mathbf{J}\right)+\gamma\left(\mathbf{I}_{\mathbf{x}}^{g} \mathbf{J}\right)^{T}\left(\mathbf{I}_{\mathbf{x}}^{g} \mathbf{J}\right)\right] } \\
&+\lambda \Psi^{\prime}\left(\rho_{Z}^{2}\right)\left(\mathbf{Z}_{\mathbf{x}} \mathbf{J}-\mathbf{K}\right)^{T}\left(\mathbf{Z}_{\mathbf{x}} \mathbf{J}-\mathbf{K}\right)+\frac{1}{2 \kappa} \mathbf{I}_{6 \times 6} .
\end{aligned}
$$


ii. For fixed $\xi=(\omega, \tau)$, compute $\chi=(\varpi, \pi)$ that minimizes:

$$
\left\{\mathbf{T V}_{c}(\pi)+\frac{\eta}{2} \sum_{\mathbf{x}}|\pi(\mathbf{x})-\tau(\mathbf{x})|^{2}\right\}+\left\{\mathbf{T V}_{\sigma}(\varpi)+\frac{\eta}{2} \sum_{\mathbf{x}}|\varpi(\mathbf{x})-\omega(\mathbf{x})|^{2}\right\}
$$

where $\eta=(\kappa \alpha)^{-1}$. Each side of equation (30) corresponds to a vectorial image denoising problem with a TV- $L^{2}$ model (ROF model). Efficient primal-dual algorithms exist for solving both problems. The left side is solved component-wise using the first-order primal-dual algorithm [3]. For the right side we use the vectorial approach [4] which allows an optimal coupling between components. A small modification is necessary in both cases to include the weighting function.

\subsection{Scene flow with camera motion estimation}

In many applications, the sensor itself moves relative to the observed scene and causes a dominant global motion in the overall motion field. In this situation, compensating for the motion of the camera can simplify the estimation and regularization of the scene flow. Moreover, for 3D reconstruction of deformable objects, the camera motion is needed to register partial 3D reconstructions. Therefore, we consider splitting the motion of the scene into a globally rigid component $\xi_{\mathrm{R}}=\left(\tau_{\mathrm{R}}, \omega_{\mathrm{R}}\right) \in \mathbb{R}^{6}$, capturing the camera motion relative to the dominant object/background, plus a non-rigid residual field $\xi=(\tau, \omega)$. We assume that a large part of the scene follows the same rigid motion. Accordingly, the scene flow is defined as the composition $\chi=\log \left(e^{\hat{\xi}}+e^{\hat{\xi}_{\mathrm{R}}}-\mathbf{I}_{4 \times 4}\right)$, and the estimation problem is formulated as:

$$
\min _{\chi} E_{\mathbf{R i g}}(\chi)+E_{\mathbf{R e s}}(\chi),
$$

with $E_{\mathbf{R i g}}(\chi)$ and $E_{\mathbf{R e s}}(\chi)$ the rigid and non-rigid energies, respectively. It is worth noting that the separation of the camera motion is in addition to the framework presented above, i.e., the non-rigid part can still deal with local motion.

Rigid energy. The camera motion can be estimated using the data term (17), by considering every pixel (or a subset of $\boldsymbol{\Omega}$ ) to solve for a unique twist $\xi_{\mathrm{R}}$. Accordingly, the rigid component of the energy is defined as:

$$
E_{\mathbf{R i g}}(\chi)=\sum_{\mathbf{x}} \Psi\left(\rho_{I}^{2}(\mathbf{x}, \chi)+\gamma \rho_{g}^{2}(\mathbf{x}, \chi)\right)+\lambda \Psi\left(\rho_{Z}^{2}(\mathbf{x}, \chi)\right)
$$

Non-rigid residual energy. The residual motion can be computed following (12), with the non-rigid energy given by:

$$
E_{\mathbf{R e s}}(\chi)=E_{D}(\chi)+\alpha E_{S}(\chi) .
$$

We minimize (31) by an iterative, alternating estimation of $\xi_{\mathrm{R}}$ and $\xi$ : 
a. Given a fixed $\xi$, solve for $\xi_{\mathrm{R}}$ that minimizes $E_{\mathbf{R i g}}(\chi)$. This is done by iteratively applying (28) and (29), with a zero auxiliary flow.

b. Given a fixed $\xi_{\mathrm{R}}$, solve for $\xi$ that minimizes $E_{\mathbf{R e s}}(\chi)$. This is done by iterating steps $\mathbf{i}$ and $\mathbf{i i}$ in Sec. 4.3.

\section{$5 \quad$ Experiments}

\subsection{Implementation details}

In order to compute the scene flow, the proposed method assumes that a pair of calibrated RGBD images is provided. Regardless of the depth sensor, depth data is always processed in cm and RGB color images are transformed to intensity images and normalized. For all the experiments $\alpha=10, \beta=1, \gamma=0.1$ and $\lambda=0.1$. For each scene a depth range is defined and only pixels having a valid depth measure inside the range are taken into account for the data term and final measurements. However, all pixels are considered in the regularization.

We use a multi-scale strategy in order to deal with larger motions. We construct an image pyramid with a downsampling factor of 2 . We apply a Gaussian anti-aliasing filter to the intensity image and the pyramid is built using bicubic downsampling. For the depth image, a $5 \times 5$ median filter is used and the pyramid is constructed by averaging pixels in non-overlapped neighborhoods of $2 \times 2$, where only pixels with a valid depth measure are used. Having a pyramid with levels $l=\{0,1, \ldots, L\}$, with 0 the original resolution, the computation is started at level $L$ an the estimated twist field is directly propagated to the next lower level. The camera matrix is scaled at each level by the factor $2^{l}$. The neighborhood $N_{\mathrm{x}}$ is defined as a $N \times N$ centering window. At each level we perform $M$ loops consisting of $M_{\mathbf{G N}}=5$ iterations of the Gauss-Newton procedure followed by $M_{\mathbf{T V}}=50$ iterations of the TV solver. The constant $\kappa$ is styled at each scale.

\subsection{Middlebury datasets}

The Middlebury stereo dataset [15] is commonly used as benchmark to compare scene flow methods $[1,8,5,13]$. Using images of one of these datasets is equivalent to a fixed camera observing an object moving in $X$ direction. As in [5, 13], we take images 2 and 6 as the first and second RGBD image, respectively, and use the ground truth disparity map of each image as depth channel. Stereobased methods $[1,8]$ do not assume RGBD images and simultaneously estimate the optical flow and disparity maps by considering images 2, 4, 6 and 8 of each dataset. The ground truth for the scene corresponds to the camera motion along the $X$ axis, while the optical flow is given by the disparity map. The scene flow error is measured in the image domain using root mean squared error (RMS) and the average angle error (AAE) of the optical flow. In order to compare with optical flow methods, we include results for the scene flow inferred using LDOF [2] and the depth data, as is described in [5]. Results and comparison for Teddy 
Table 1. Middlebury dataset: errors on the optical flow extracted from the scene flow (except for [2], which is an optical flow method). See Sec. 5.2 for details.

\begin{tabular}{|c|c|c|c|c|c|}
\cline { 3 - 6 } \multicolumn{2}{c}{} & \multicolumn{2}{c|}{ Teddy } & \multicolumn{2}{c|}{ Cones } \\
\cline { 2 - 6 } \multicolumn{1}{c|}{} & Views & RMS & AAE & RMS & AAE \\
\hline Semi-rigid Scene Flow (ours) & 1 & 0.49 & 0.46 & 0.45 & 0.37 \\
\hline Hadfield and Bowden [5] & 1 & 0.52 & 1.36 & 0.59 & 1.61 \\
\hline Quiroga et al. $[13]$ & 1 & 0.94 & 0.84 & 0.79 & 0.52 \\
\hline Brox and Malik [2]+ depth & 1 & 2.11 & 0.43 & 2.30 & 0.52 \\
\hline Basha et al. $[1]$ & 2 & 0.57 & 1.01 & 0.58 & 0.39 \\
\hline Huguet and Devernay [8] & 2 & 1.25 & 0.51 & 1.10 & 0.69 \\
\hline
\end{tabular}

and Cones datasets are shown in Table 1, where stereo methods are denoted with 2 views. In this experiment, we used a 5-level pyramid, with $M=5, N=3$ and for each level $l$, we set $\kappa=10^{4} 10^{-l}$. A non-optimized implementation of our method processes each dataset in about $60 \mathrm{sec}$. The proposed approach outperforms previous methods. Because the $3 \mathrm{D}$ motion field resulting from camera translation is constant, Middlebury datasets are not well suited to fully evaluate the performance of scene flow methods.

\subsection{Scene flow from RGBD data}

We performed further experiments on more complex scenes using two RGBD sensors: the Microsoft Kinect for Xbox and the Asus Xtion Pro Live. We consider three different setups: i) a fixed camera, ii) a moving camera observing a rigid scene and iii) a moving camera capturing deformable objects. In each case, we show the input images, the optical flow, and one or more components of the scene flow. To give an idea of the motion, the average of the two input RGB images is shown. The optical flow is visualized using the Middlebury color code [15]. For the scene flow, we show each component using a cold-to-warm code, where green color is zero motion, and warmer and colder colors represent positive and negative velocities, respectively.

In the first experiment, the Kinect sensor is fixed to compute the scene flow from two sequences (Fig. 1). The top row shows the deformation of a poster, which produces a non-uniform deformation. The proposed method is able to capture the poster deformation, thus it is possible to accurately estimate the changes in depth when the poster is folded. The gradient constancy constraint plays an important role here, since the sensor applies automatic white balancing. The bottom images show a motion performed with arms and hands. While hands are rotating inwards, the elbows lift, and a region of both arms remains almost still. This composite motion generates a discontinuous optical flow, which is well estimated by our method. Moreover, it can be seen that small rotations and articulated motions are well described for the proposed motion model.

The second experiment considers a static scene observed by a moving camera. We use Teddy images of the RGBD dataset [17]. Unlike the Middlebury dataset, 

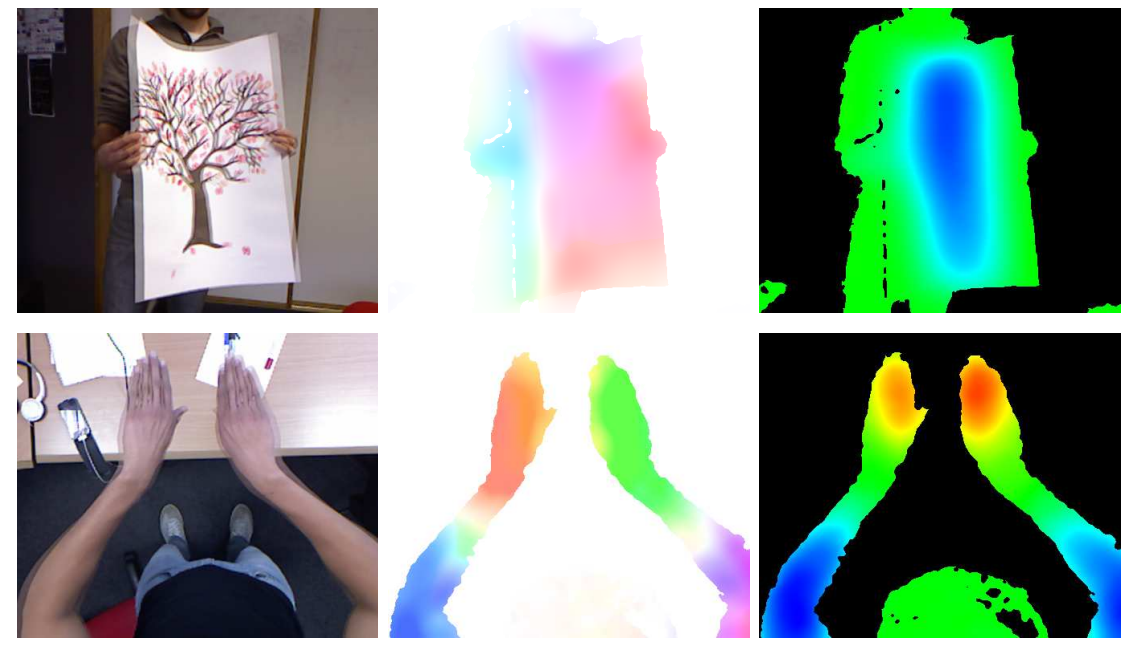

Fig. 1. Scene flow estimation with a fixed camera. a) Left: Input images. b) Middle: Optical flow c) Right: Z-component of the scene flow.
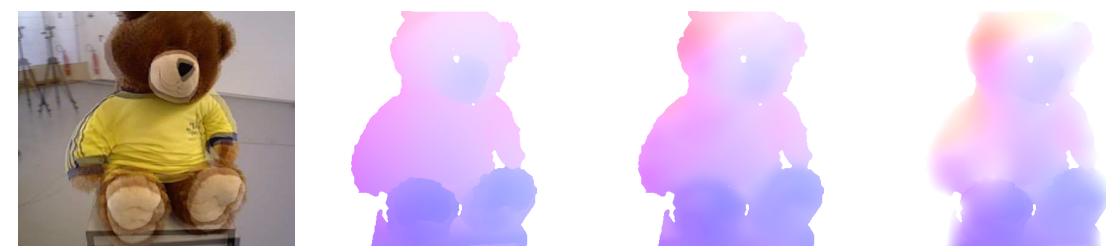

Fig. 2. Camera motion. From left to the right: a) Input images. b) Optical flow of the rigid motion estimation. c) Optical flow using the proposed method. d) Optical flow by the scene flow method [13]. Results of the proposed method are clearly more accurate than those of [13].

this scene presents a changing 3D motion field due to the translation and rotation of the sensor. We first estimate a single rigid motion as is presented in Sec. 4.4. We estimate the parameters using every second pixel with a range between $50 \mathrm{~cm}$ and $150 \mathrm{~cm}$ from the sensor. At each level of the pyramid we run 100 iterations. This result is taken as baseline and compared in Figure 2 with resulting optical flows of our method and the dense estimation provided by [13]. The proposed method produces a close estimation of the camera motion without assuming a global rigidity of the scene. In contrast, the direct regularization on the scene flow components, as is performed in [13], fails to capture the diversity of $3 \mathrm{D}$ motions introduced by the camera rotation.

In the last experiment, a moving Asus Xtion camera observes a non-rigid scene. To estimate the rigid and non-rigid components we perform 3 rounds of 

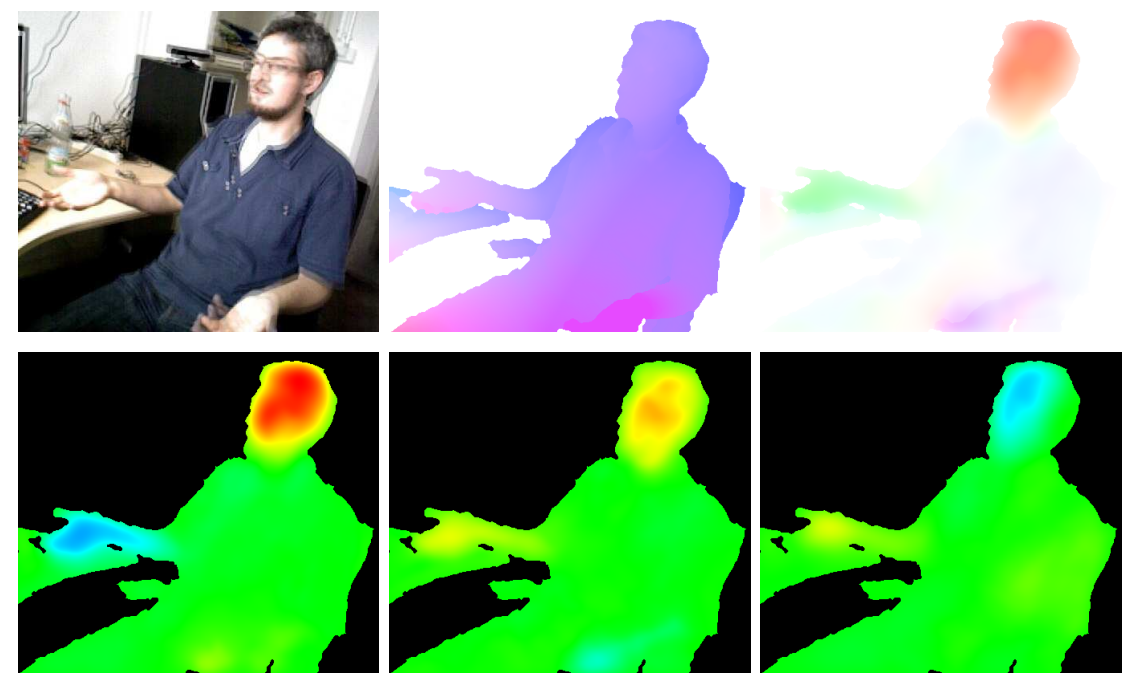

Fig. 3. Motion estimation with a moving camera. Top, from left to right: a) Input images. b) Optical flow of the global rigid motion. c) Optical flow of the non-rigid residual. Bottom, from left to right: $(X, Y, Z)$ components of the non-rigid 3D motion.

alternation at each level of the pyramid. As is shown in Figure 3, the proposed approach allows the joint estimation of both components. Particularly, it is possible to compute the motion of both hands and the rotation of the face while the camera is turning. Capturing the motion of thin objects, as the fingers of the left hand, is a challenge since depth data is incomplete and very noisy in this area.

The size of the local neighborhood was kept fixed for all the scales and for every position on the image. Results could be improved by adjusting the size of the window using the depth data, in order to get a constant resolution.

\section{Summary}

We have presented a new method to compute dense scene flow from RGBD images by modeling the motion as a field of rigid motions. This allows for piecewise smooth solutions using TV regularization on the parametrization. We have decoupled the regularization procedure for the rotational and translational part and proposed some approximations to simplify the optimization. Future advances on manifold regularization may provide even more accurate and faster solvers that can be used with our parameterization. In order to fully exploit both intensity and depth data, we constrain the rigid body motion in the image domain. This way, we can solve for the local rigid motion as an iteratively reweighted least squares problem. The proposed approach provides an adjustable combination between local and piecewise rigidity, which, in conjunction with a global rigid estimation, is able to capture the motion in real world scenes. 


\section{References}

1. Basha, T., Moses, Y., Kiryati, N.: Multi-view scene flow estimation: A view centered variational approach. In: Conference on Computer Vision and Pattern Recognition. pp. 1506-1513 (2010)

2. Brox, T., Malik, J.: Large displacement optical flow: Descriptor matching in variational motion estimation. IEEE Transactions on Pattern Analysis and Machine Intelligence 33(3), 500-513 (2011)

3. Chambolle, A., Pock, T.: A first-order primal-dual algorithm for convex problems with applications to imaging. Journal of Mathematical Imaging and Vision 40(1), 120-145 (2011)

4. Goldluecke, B., Strekalovskiy, E., Cremers, D.: The natural vectorial total variation which arises from geometric measure theory. SIAM Journal on Imaging Sciences 5(2), 537-563 (2012)

5. Hadfield, S., Bowden, R.: Scene particles: Unregularized particle-based scene flow estimation. IEEE Transactions on Pattern Analysis and Machine Intelligence 36(3), 564-576 (2014)

6. Herbst, E., Ren, X., Fox, D.: RGB-D flow: Dense 3-D motion estimation using color and depth. In: International Conference on Robotics and Automation (ICRA). pp. 2276-2282 (2013)

7. Horn, B.K., Schunck, B.G.: Determining optical flow. Artificial Intelligence 17, 185-203 (1981)

8. Huguet, F., Devernay, F.: A variational method for scene flow estimation from stereo sequences. In: International Conference on Computer Vision. pp. 1-7 (2007)

9. Kerl, C., Sturm, J., Cremers, D.: Robust odometry estimation for RGB-D cameras. In: International Conference on Robotics and Automation (ICRA). pp. 3748-3754 (2013)

10. Letouzey, A., Petit, B., Boyer, E.: Scene flow from depth and color images. In: British Machine Vision Conference (BMVC), 2011 (2011)

11. Murray, R.M., Sastry, S.S., Zexiang, L.: A Mathematical Introduction to Robotic Manipulation. CRC Press, Inc., Boca Raton, FL, USA, 1st edn. (1994)

12. Quiroga, J., Devernay, F., Crowley, J.: Scene flow by tracking in intensity and depth data. In: Computer Vision and Pattern Recognition Workshops (CVPRW). pp. 50-57 (2012)

13. Quiroga, J., Devernay, F., Crowley, J.: Local/global scene flow estimation. In: International Conference on Image Processing (ICIP). pp. 3850-3854 (2013)

14. Rosman, G., Bronstein, A., Bronstein, M., Tai, X.C., Kimmel, R.: Group-valued regularization for analysis of articulated motion. In: European Conference on Computer Vision Workshops. pp. 52-62 (2012)

15. Scharstein, D., Szeliski, R.: High-accuracy stereo depth maps using structured light. In: Conference on Computer Vision and Pattern Recognition. pp. 195-202 vol.1 (2003)

16. Spies, H., Jahne, B., Barron, J.: Dense range flow from depth and intensity data. In: International Conference on Pattern Recognition. pp. 131-134 vol.1 (2000)

17. Sturm, J., Engelhard, N., Endres, F., Burgard, W., Cremers, D.: A benchmark for the evaluation of RGB-D slam systems. In: International Conference on Intelligent Robot Systems (IROS). pp. 573-580 (2012)

18. Vedula, S., Baker, S., Rander, P., Collins, R.: Three-dimensional scene flow. In: International Conference on Computer Vision. pp. 722-729 vol.2 (1999) 
19. Vogel, C., Schindler, K., Roth, S.: 3D scene flow estimation with a rigid motion prior. In: International Conference on Computer Vision. pp. 1291-1298 (2011)

20. Wang, Y., Yang, J., Yin, W., Zhang, Y.: A new alternating minimization algorithm for total variation image reconstruction. SIAM J. Img. Sci. 1(3), 248-272 (2008)

21. Wedel, A., Rabe, C., Vaudrey, T., Brox, T., Franke, U., Cremers, D.: Efficient dense scene flow from sparse of dense stereo data. In: European Conference on Computer Vision. pp. 739-751 (2008)

22. Wedel, A., Pock, T., Zach, C., Bischof, H., Cremers, D.: An improved algorithm for TV-L1 optical flow. In: Cremers, D., Rosenhahn, B., Yuille, A., Schmidt, F. (eds.) Statistical and Geometrical Approaches to Visual Motion Analysis, Lecture Notes in Computer Science, vol. 5604, pp. 23-45. Springer Berlin Heidelberg (2009)

23. Werlberger, M., Trobin, W., Pock, T., Wedel, A., Cremers, D., Bischof, H.: Anisotropic Huber-L1 Optical Flow. In: Proceedings of the British Machine Vision Conference (BMVC) (2009) 